\title{
Analysis of 3208 cystic fibrosis prenatal diagnoses: Impact of carrier screening guidelines on distribution of indications for CFTR mutation and IVS-8 poly(T) analyses
}

Elizabeth M. Rohlfs, $P h D^{1}$, Vivian J. Weinblatt, $M S^{2}$, Karen J. Treat, $M S^{1}$, and Elaine A. Sugarman, $M S^{1}$

\begin{abstract}
Purpose: To evaluate and quantify indications for CFTR mutation analysis of prenatal specimens, and to determine if a significant portion of tests are performed only for the identification of $5 \mathrm{~T}$ alleles, we surveyed our laboratory data over a 3-year time period that spanned the issuance of the cystic fibrosis (CF) carrier screening guidelines. Methods: Referral indications for 3208 prenatal specimens were compared for an 18-month period before (April 2000 to September 2001) and after (October 2001 to April 2003) publication of the ACMG/ACOG statement regarding prenatal and preconception testing for CF. Results: The frequency of cases received for testing when one or both parents were CF mutation carriers did not change significantly after publication of the guidelines. The most frequent indication during the entire 3-year period was fetal ultrasound abnormality, yet in the post-ACMG/ACOG period the percentage decreased significantly due to an increase in the number of prenatal screening cases. Testing indications related to parental 5T status also increased significantly in the post-ACMG/ACOG period and accounted for $2.9 \%$ of testing over the 3-year period. A small subset (1.6\%) of prenatal specimens were tested for poly $(\mathrm{T})$ even though the parents did not carry $5 \mathrm{~T}$ allele(s). However, more than $40 \%$ of these cases could be attributed to parental R117H mutations. Conclusion: These data indicate that although indications for prenatal testing shifted after the issuance of carrier screening guidelines, prenatal testing related to parental 5T alleles comprised $<3 \%$ of the total referral indications. Genet Med 2004:6(5):400-404.
\end{abstract}

Key Words: CFTR mutations, prenatal testing, $5 T$ allele, IVS-8 poly(T) variant

Cystic fibrosis (CF) is one of the most common autosomal recessive disorders in Caucasian populations. Although the average lifespan of individuals affected with CF has risen to approximately 33 years of age, the disease is characterized by progressive lung disease due to chronic infection, pancreatic exocrine insufficiency, infertility in males, and elevated sweat chloride levels. ${ }^{1,2}$ Over the last decade, mutation analysis of the CFTR gene in patients with CF and related conditions, has identified more than 1300 mutations. ${ }^{3}$ Prenatal diagnoses for CF became available with the identification of the gene and offered choices not previously available to couples with a 1 in 4 risk of having a child with CF. Testing the fetus for CF mutations also has utility when fetal echogenic bowel is identified. ${ }^{4-6}$ There is limited data available on the frequency of other CF prenatal diagnosis indications and the effects of the institution of carrier screening guidelines. ${ }^{7}$

\footnotetext{
From the ${ }^{1}$ Genzyme Corporation, Genzyme Genetics, Molecular Diagnostic Laboratory Westborough, Massachusetts; ${ }^{2}$ Genzyme Genetics, Philadelphia, Pennsylvania.

Elizabeth Rohlfs, PhD, Genzyme Genetics, Molecular Diagnostic Laboratory, 3400 Computer Drive, Westborough, MA 01518.

Received: May 3, 2004.

Accepted: June 28, 2004.
}

DOI: 10.1097/01.GIM.0000139504.05850.4B
In 2001, the American College of Medical Genetics (ACMG), American College of Obstetricians and Gynecologists (ACOG), and $\mathrm{NIH}$ collaborated to publish guidelines for CF screening in the general population. ${ }^{8,9}$ The guidelines acknowledge that screening is most effective when performed before pregnancy and offer guidance for prenatal diagnosis when both parents carry a $\mathrm{CF}$ mutation. They also recommend that laboratories offering CF screening include a minimum of 25 specific mutations in their panel, with additional mutations included if warranted by the local demographics. ${ }^{10,11}$

Included in the ACMG/ACOG 25 mutation panel is R117H, a mutation known to have variable phenotypic expression. When $\mathrm{R} 117 \mathrm{H}$ is identified during carrier screening, the guidelines recommend additional testing to determine the length of the intron 8 polythymidine tract $(\operatorname{poly}(\mathrm{T}))$. When $\mathrm{R} 117 \mathrm{H}$ is found in cis with 5 thymidines (5T), and trans to a severe CF mutation, individuals may have moderate (i.e., pancreatic sufficient) CF. When R117H is identified in cis with 7 thymidines (7T) and in trans to a CF mutation individuals may be asymptomatic, have congenital absence of the vas deferens (CAVD), or later onset lung disease (i.e., a milder phenotype). ${ }^{12,13}$

In addition to being identified on the same chromosome as $\mathrm{R} 117 \mathrm{H}$, the $5 \mathrm{~T}$ allele occurs alone in approximately $10 \%$ of the general population. ${ }^{12}$ When the $5 \mathrm{~T}$ allele is included in CF 
screening panels, many individuals are identified who are not at risk for having a child with classic CF. ${ }^{7}$ Identification of $5 \mathrm{~T}$ carrier status complicates genetic counseling because it is not possible to assess a risk for each of the possible phenotypic outcomes of various genotypes involving 5T. A 5T allele in trans to a CF mutation may be associated with a number of clinical presentations including, no symptoms, ${ }^{14}$ CAVD in males, ${ }^{15,16}$ chronic pancreatitis, ${ }^{17}$ or atypical or typical CF. ${ }^{18}$ Also, two copies of the $5 \mathrm{~T}$ allele have been identified in healthy individuals, ${ }^{14}$ men with CAVD, ${ }^{19}$ and in persons with CF-like lung disease, ${ }^{20}$ or bronchiectasis ${ }^{21}$ and no other identified CF mutations. This range of possible phenotypic outcomes has resulted in some couples choosing prenatal diagnoses for conditions not intended by the CF screening guidelines. ${ }^{22}$

Recent reports in both the scientific and lay literature have suggested that there are a substantial number of couples choosing to undergo invasive prenatal procedures solely due to the presence of a $5 \mathrm{~T}$ allele in one or both parents..$^{22-27}$ However, the indications for CF prenatal diagnoses, including poly $(\mathrm{T})$ testing, have yet to be quantified. As emphasized in a recent editorial, evaluation of such data are necessary to assess the impact and performance of the CF screening guidelines. ${ }^{28}$ We surveyed indications for prenatal diagnosis for CF over a 3-year time period. These data indicate that although indications for prenatal testing shifted after the issuance of carrier screening guidelines, prenatal testing related to parental $5 \mathrm{~T}$ alleles comprises $<3 \%$ of referral indications.

\section{MATERIALS AND METHODS}

\section{Patient samples}

From April 2000 through April 2003, 3208 prenatal samples (amniotic fluid, chorionic villi, or cultured cells from amniotic fluid or chorionic villi) were received in our laboratory for CFTR mutation analysis. In the 18-month period (April 2000 to September 2001) before the publication of the CF carrier screening guidelines (pre-ACMG/ACOG), 1330 fetal specimens were received and tested. In the 18-month period (October 2001 to April 2003) after the publication of the guidelines (post-ACMG/ACOG), 1878 fetal specimens were received and tested. Information regarding the indication for prenatal CF testing was provided by the referring physician.

\section{CFTR mutation analysis}

Genomic DNA was isolated from all specimen types using standard extraction methods. All samples were tested for 87 CFTR mutations by a pooled allele-specific hybridization strategy method described previously. ${ }^{29,30}$ Nineteen regions of the CFTR gene were amplified in two multiplex polymerase chains reactions (PCR). The amplified PCR products were immobilized on positively charged nylon membrane and hybridized with groups of radioactively labeled ASO probes. Individual mutation identification of pool-positive samples was made by individual ASO hybridization to normal and mutant alleles.

CFTR intron 8 poly $(\mathrm{T})$ variant analysis was performed on a subset of 139 (4.3\%) of these prenatal specimens. Analysis was performed by PCR amplification of a region of intron 8 and exon 9 spanning the poly(T) tract. The length of the poly $(\mathrm{T})$ tract was determined using an allele-specific hybridization method specific for the $5 \mathrm{~T}, 7 \mathrm{~T}$, or $9 \mathrm{~T}$ alleles.

\section{Statistical analysis}

The difference between the frequency of prenatal test indications pre- and post-ACMG/ACOG was tested by the Chisquare test. A value of $P<0.05$ was considered to indicate statistical significance.

\section{RESULTS}

\section{Indications for prenatal CFTR testing}

When prenatal testing indications are examined over the entire period from April 2000 through April 2003, the fre-

Table 1

Indication categories for prenatal CFTR testing

\begin{tabular}{|c|c|c|c|c|}
\hline Indication & $\begin{array}{c}\text { Pre-ACMG/ACOG } \\
n=1330(\%)\end{array}$ & $\begin{array}{c}\text { Post-ACMG/ACOG } \\
n=1878(\%)\end{array}$ & $\begin{aligned} & \text { Total } \\
n= & 3208(\%)\end{aligned}$ & $P$ value $^{a}$ \\
\hline Unrelated to $5 \mathrm{~T}$ status & $1310(98.5)$ & $1806(96.2)$ & $3116(97.1)$ & 0.0002 \\
\hline Abnormal fetal ultrasound & $656(49.3)$ & $576(30.7)$ & $1232(38.4)$ & 0.0001 \\
\hline Both parents CF carriers & $198(14.9)$ & $277(14.7)$ & $475(14.8)$ & $\mathrm{ns}^{b}$ \\
\hline One parent CF carrier or affected with CF & $261(19.6)$ & $341(18.2)$ & $602(18.8)$ & $\mathrm{ns}^{b}$ \\
\hline Unspecified family history of CF & $54(4.1)$ & $45(2.4)$ & $99(3.1)$ & 0.0098 \\
\hline Prenatal screening & $141(10.8)$ & $567(30.2)$ & $708(22.1)$ & 0.0001 \\
\hline Related to $5 \mathrm{~T}$ status & $20(1.5)$ & $72(3.8)$ & $92(2.9)$ & 0.0002 \\
\hline Fetus at risk for $5 \mathrm{~T}$ and $\mathrm{CF}$ mutation in trans & $9(0.7)$ & $39(2.0)$ & $48(1.5)$ & 0.0021 \\
\hline One or both parents positive for only $5 \mathrm{~T}$ & $11(0.8)$ & $33(1.8)$ & $44(1.3)$ & $0.0378^{a}$ \\
\hline
\end{tabular}

${ }^{a}$ Chi-square test for comparison of frequency of indication in pre- and post-ACMG/ACOG time periods.

${ }^{b}$ Not significant. 
quency of cases received for testing when one or both parents were CF mutation carriers did not change significantly after publication of the guidelines (Table 1). Almost $15 \%$ of the cases were from couples with a 1 in 4 risk of having a child with $\mathrm{CF}$. A slightly greater percentage of cases were from couples where only one parent was known to carry a CF mutation(s). There was a significant decrease in the frequency of cases related to an unspecified family history of CF. Overall the most frequent indication was abnormal fetal ultrasound findings $(38.4 \%)$. In the majority (95.3\%) of cases in this category, parental CF mutation status was not known at the time of fetal testing. The frequency of cases referred due to abnormal ultrasound findings decreased significantly in the post-ACMG/ ACOG period. In contrast, the frequency of prenatal screening with no increased risk factors for CF, increased from $10.8 \%$ to $30.2 \%(P=0.0001)$ during the same period. These indications, which are unrelated to parental $5 \mathrm{~T}$ allele status, accounted for $>97 \%$ of referrals for prenatal CFTR analysis.

Further examination of the specimens referred for prenatal CF screening without a known increased risk for CF showed that $87 \%$ of the mothers were $>34$ years of age at the time of testing. This suggests that their motivation to undergo a prenatal procedure may have been influenced by advanced maternal age. Of the remaining cases, an additional $6 \%$ were between 30 and 34 years of age and $7 \%$ were $<30$ years of age at the time of testing. One CFTR mutation was detected in $5 \%$ of the prenatal specimens that were screened.

Indications for prenatal CFTR testing related to $5 \mathrm{~T}$ status include the following: (1) fetus at risk for inheriting a $5 \mathrm{~T}$ allele and CFTR mutation in trans, and (2) one or both parents positive for only $5 \mathrm{~T}$ allele(s). When one parent carried a CF mutation and the other a 5T allele, we were not able to determine if the identification of a $5 \mathrm{~T}$ during screening caused the other parent to be screened with subsequent identification of the $\mathrm{CF}$ mutation or vice versa. In the post-ACMG/ACOG period, the number of prenatal cases increased in both indication categories (Table 1). Among those fetuses at risk for one copy of $5 \mathrm{~T}$ and a CFTR mutation, the frequency increased from $0.7 \%$ to $2.0 \%(P=0.0021)$. In the second category, the percentage also increased from $0.8 \%$ to $1.8 \%(P=0.0378)$. Overall there were
92 (2.9\% of total) specimens received for testing when one or both parents were previously known to carry a $5 \mathrm{~T}$ allele.

\section{Poly(T) testing of prenatal specimens}

Our laboratory offers poly $(\mathrm{T})$ testing separately from CFTR mutation analysis. However, in order to provide an appropriate interpretation of poly $(\mathrm{T})$ results, analysis for CFTR mutations is also required. The frequency of requests for poly $(\mathrm{T})$ variant testing of prenatal specimens according to indication were also analyzed (Table 2 ). When the parents did not carry a $5 \mathrm{~T}$ allele, $1.6 \%$ of prenatal specimens were also tested for poly $(\mathrm{T})$. A significant number of these tests could be attributed to parental $\mathrm{R} 117 \mathrm{H}$ mutations. More than $50 \%$ of those cases with a family history of CF or a CF carrier that were tested for poly $(\mathrm{T})$, were at risk for inheriting an $\mathrm{R} 117 \mathrm{H}$ mutation. The two prenatal screening cases that were also tested for poly $(\mathrm{T})$, were done so after identification of $\mathrm{R} 117 \mathrm{H}$ in the fetus. As expected, among those specimens whose indication for testing included specific mention of parental 5T carrier status, poly $(\mathrm{T})$ testing was always ordered.

\section{Poly $(T)$ results in prenatal testing}

Testing of 139 fetal specimens for both CFTR and poly(T) mutation analyses identified seven fetuses that were homozygous for $5 \mathrm{~T}$ and 12 fetuses that were positive for a CFTR mutation and $5 \mathrm{~T}$ allele in trans. According to the referring health care providers, five of the seven homozygous $5 \mathrm{~T}$ fetuses resulted in delivery of healthy infants. Followup information was unavailable on the remaining two. Among the 12 fetuses identified with a CF mutation and 5T in trans, 7 apparently healthy infants were delivered, one infant died secondary to an unbalanced chromosomal translocation and multiple congenital anomalies, and one infant experienced respiratory distress syndrome secondary to prematurity but had a negative sweat test. Followup information was not available on the remaining three fetuses. Whereas detailed clinical evaluation of these infants was not available, severe symptoms were not reported but mild symptoms may not yet have been evident and later onset disease cannot be ruled out.

Table 2

Indication categories for prenatal Poly(T) testing

\begin{tabular}{lccc}
\hline Indication & No. of cases (\%) & No. requesting poly(T) testing (\%) & No. with R117H carrier parent (\%) \\
\hline Unrelated to 5T status & $3116(97.1)$ & $47(1.6)$ & $10(0.8)$ \\
Abnormal fetal ultrasound & $1232(38.4)$ & $35(3.0)$ & $2(20.0)$ \\
Family history of CF or CF carrier & $1176(36.7)$ & $2(0.3)$ & $18(51.4)$ \\
Prenatal screening & $708(22.0)$ & $92(100)$ & 0 \\
Related to 5T status & $92(2.9)$ & $48(100)$ & $7(7.6)$ \\
$\quad$ Fetus at risk for 5T and CF mutation in trans & $48(1.5)$ & $44(100)$ & $7(14.6)$ \\
One or both parents positive for only 5T & $44(1.4)$ & $139(4.3)$ & $27(19.4)$ \\
All indications & 3208 & & \\
\hline
\end{tabular}




\section{DISCUSSION}

Our purpose was to determine the frequency of specific indications for prenatal CFTR and poly(T) testing and to determine if the ACMG/ACOG CF screening statement influenced the frequency of these requests. To accomplish this, we analyzed requests for CFTR and poly(T) testing of prenatal specimens for the 18 months preceding the October 2001 statement and the 18 months following the statement. Over the entire study period, the largest referral indication was fetal ultrasound abnormality, which is appropriate due to the association between CF and echogenic bowel. ${ }^{4-6}$ Approximately $15 \%$ of prenatal specimens were tested because both parents carried a CF mutation and had a 1 in 4 risk for an affected child. This percentage remained stable over the 3-year period. A slightly greater percentage of cases were referred when one parent carried a CF mutation. Even though the risk to have an affected child is less, lack of carrier information from the other parent may make testing the fetus a likely course of action for some patients. The other parent may not be tested due to unavailability or third party payer policies regarding reimbursement.

We also observed a relatively high rate of referrals for prenatal screening of fetuses with no family history of CF. In addition, the rate of referrals increased significantly in the postACMG/ACOG period. Further examination of the patient information showed that $87 \%$ of these women were over 34 years of age. We suspect that these women were not screened for CF preconceptionally and a prenatal procedure was being independently performed for routine chromosome analysis. Other possible reasons for screening prenatal specimens for $\mathrm{CF}$ again include the unavailability of one or both parents for screening and reimbursement concerns. CF screening via amniocentesis and CVS does not address CF risk in subsequent pregnancies, and should not be considered the preferred method for general population screening. Additionally, when a mutation is detected during prenatal screening both parents must be tested in order to fully assess the risk for the fetus to be affected. This may result in increased anxiety at a late stage of pregnancy as testing is completed.

We found that overall the majority of prenatal testing indications $(>97 \%)$ were for reasons unrelated to poly $(\mathrm{T})$ status. However, indications related to parental $5 \mathrm{~T}$ status increased $(1.5 \%$ to $3.8 \%)$ in the post-ACMG/ACOG period, which is most likely due to increased identification of $5 \mathrm{~T}$ alleles during carrier testing. Identification of $5 \mathrm{~T}$ by screening for poly $(\mathrm{T})$ alleles presents challenges for health care providers because of the range of diverse and unpredictable phenotypes. As a result, the ACMG/ACOG guidelines specifically state that screening for $5 \mathrm{~T}$ alleles should only be performed when the $\mathrm{R} 117 \mathrm{H} \mathrm{mu}$ tation is identified. Inappropriate carrier testing for $5 \mathrm{~T}$ alleles should be avoided because it may result in testing of prenatal specimens for conditions not intended by the CF screening guidelines.

Our data also demonstrate that poly(T) analysis is infrequently ordered as a part of routine prenatal testing for CF. When all cases, regardless of indication, are considered, $4.3 \%$ of prenatal specimens received for CFTR testing include poly $(\mathrm{T})$ testing. When the cases that include an indication of parental $5 \mathrm{~T}$ alleles are not included in the analysis, the percentage requesting poly $(\mathrm{T})$ testing drops to $1.6 \%$. When those cases related to an $\mathrm{R} 117 \mathrm{H}$ mutation are excluded (i.e., parental or fetal $\mathrm{R} 117 \mathrm{H}$ ), the percentage drops further to $0.8 \%$. Providing poly $(\mathrm{T})$ information in the context of an $\mathrm{R} 117 \mathrm{H}$ mutation is recommended by the ACMG/ACOG carrier screening guidelines and is necessary and appropriate for the genetic counseling process.

Because we observed that health care providers order poly $(\mathrm{T})$ testing when fetuses are at risk for inheriting two copies of 5T or a 5T and CF mutation in trans, we sought to obtain follow up clinical information. Fourteen of nineteen infants that were diagnosed prenatally with the above genotypes could be identified to followup. None had recognized symptoms of classic CF. The challenge of risk analysis and family counseling continues, however, as mild symptoms or later onset disease cannot be ruled out for these patients.

In summary, we find that the frequency of prenatal referral indications shifted after publication of the CF carrier screening guidelines and that poly $(\mathrm{T})$ testing is not routinely ordered on prenatal specimens unless one of the parents has been previously identified with a $5 \mathrm{~T}$ allele or carried an R117H mutation. As expected, followup of 5T-positive pregnancies revealed no evidence of classic CF, but long-term followup is required to further define the 5T phenotypes. Finally, the observed rates of prenatal screening for CF in low-risk populations, emphasize a need for further education regarding appropriate indications for prenatal CFTR testing and the advantages of parental carrier testing before prenatal diagnosis.

\section{ACKNOWLEDGMENTS}

We are thankful for the contributions of Nichole Napolitano, Joanne Garver, and the technical and clinical staff of the Molecular Diagnostic Laboratory at Genzyme Genetics who are responsible for assay performance and technical and clinical review of patient results.

\section{References}

1. Cystic Fibrosis Foundation. Available at: http://www.cff.org. Accessed on June 20, 2004.

2. Welsh MJ, Ramsey BW, Accurso F, Cutting GR. Cystic Fibrosis. In: Scriver CR, Beaudet AL, Sly WS, Valle D et al, editors. Metabolic and Molecular Bases of Inherited Disease. New York: McGraw-Hill; 2001:5121-5188.

3. Cystic Fibrosis Mutation Data Base. Available at: http://www.genet.sickkids.on.ca/ CFTR/. Accessed on June 20, 2004

4. Muller F, Simon-Bouy B, Girodon E, Monnier N, Malinge MC, Serre JL and the French Collaborative Group. Predicting the risk of cystic fibrosis with abnormal ultrasound signs of fetal bowel: Results of a French Molecular Collaborative Study based on 641 prospective cases. Am J Med Genet 2002;110:109-115.

5. Slotnick RN, Abuhamad AZ. Prognostic implications of fetal echogenic bowel. Lancet 1996:347;85-87.

6. Monaghan KG, Feldman GL. The risk of cystic fibrosis with prenatally detected echogenic bowel in an ethnically and racially diverse North American population. Prenat Diag 1999;19:609-609.

7. Strom CM, Crossley B, Redman JB, Buller A, Quan F, Meng M et al. Cystic fibrosis screening: Lessons learned from the first 320,000 patients. Genet Med 2004;6:136140 . 


\section{Rohlfs et al.}

8. Grody WW, Cutting GR, Klinger KW, Richards CS, Watson MS, Desnick RJ. Laboratory standards and guidelines for population-based cystic fibrosis carrier screening. Genet Med 2001;3:456-461.

9. ACOG. 2001. Preconception and prenatal carrier screening for CF: Clinical and laboratory guidelines. American College of Obstetricians and Gynecologists, Washington, D.C.

10. Richards CS, Bradley LA, Amos J, Allitto BA, Grody WW, Maddelena A et al. Standards and guidelines for CFTR mutation testing. Genet Med 2002;4(5):379-391.

11. Watson M, Cutting GR, Desnick RJ, Driscoll DA, Klinger K, Mennuti M et al. Cystic Fibrosis (CF) Couple Carrier Screening: 2004 Revision of American College of Medical Genetics Mutation Panel. Genet Med 2004;6:387-391.

12. Kiesewetter S, Macek M, Davis C, Curristin SM, Chu C-S, Graham C et al. A mutation in CFTR produces different phenotypes depending on chromosomal background. Nat Genet 1993;5:274-278.

13. Massie RJH, Poplawski N, Wilcken B, Goldblatt J, Byrnes C, Robertson C. Intron-8 polythymdine sequence in Australian individuals with CF mutations $\mathrm{R} 117 \mathrm{H}$ and R117C. Eur Respir J 2001;17:1195-1200.

14. Rave-Harel N, Kerem E, Nissim-Rafina M, Madjar I, Goshen R, Augarten A et al. The molecular basis of partial penetrance of splicing mutations in cystic fibrosis. Am J Hum Genet 1997;60:87-94.

15. Chillon M, Casals T, Mercier B, Bassas L, Lissens W, Silber S et al. Mutations in the cystic fibrosis gene in patients with congenital absence of the vas deferens. $N$ Engl J Med 1995;332:1475-1480.

16. Lissens W, Mercier B, Tournaye H, Bonduelle M, Ferec C, Seneca S et al. Cystic fibrosis and infertility caused by congenital absence of the vas deferens and related clinical entities. Hum Reprod 1996;11:55-80.

17. Noone PG, Knowles MR. "CFTR -opathies": disease phenotypes associated with cystic fibrosis transmembrane conductance regulator gene mutations. Respir Res 2001;2:328-332.

18. Kerem E, Rave-Harel N, Augarten A, Madgar I, Nissim-Rafina M, Yahav Y et al. A cystic fibrosis transmembrane conductance regulator splice variant with partial penetrance associated with variable cystic fibrosis presentation. Am J Respir Crit Care Med 1997;155:1914-1920.
19. Dork T, Dworniczak B, Aulehla-Scholz C, Wieczorek D, Bohm I, Mayerova A, Seydewitz $\mathrm{HH}$ et al. Distinct spectrum of CFTR gene mutations in congenital absence of the vas deferens. Hum Genet 1997;100:365-377.

20. Noone PG, Pue CA, Zhou Z, Friedman KJ, Wakelink EL, Ganeshananthan M et al. Lung disease associated with the IVS8 5T allele of the CFTR gene. Am J Respir Crit Care Med 2000;162:1919-1924.

21. Pignatti PF, Bombieri C, Benetazzo M, Casartelli A, Trabetti E, Gile LS et al. CFTR gene variant IVS8-5T in disseminated bronchiectasis. Am J Hum Genet 1996;58: $889-892$.

22. Redman J, Franklin CR, LeGrave D, York M, Entwistle TD, Strom CM. Reporting the CFTR intron $85 \mathrm{~T}$ allele in general population screening: muddying the waters. Genet Med 2003;5:223.

23. Hudson K. A warning that must not be ignored. New Scientist May 3, 2003.

24. Concar D. Genetic test blunders risk needless abortions. New Scientist April 30, 2003.

25. Zitner A. Lost in translation. Gene testing is proving to be a challenge: In one broad screening, results have been difficult to interpret, creating unintended risks. Los Angeles Times May 12, 2003.

26. Vastag B. Cystic fibrosis gene testing a challenge. JAMA 2003;289:2923-2924.

27. Bell J Cystic Fibrosis tests raise alarm. Concern: Evidence suggests doctors misinterpret results from couples, leading to unnecessary prenatal procedures. Baltimore Sun May 19, 2003.

28. Palomaki GE. Prenatal screening for cystic fibrosis: An early report card. Genet Med 2004;6:115-116

29. Heim RA, Sugarman EA, Allitto BA. Improved detection of cystic fibrosis mutation in the heterogeneous U.S. population using an expanded pan-ethnic mutation panel. Genet Med 2001;3:168-176.

30. Shuber A, Skoletsky J, Stern R, Handelin B. Efficient 12-mutation testing in the CFTR gene: A general model for complex mutation analysis. Hum Mol Genet 1993; 2:153-158. 